\title{
The Amplitude-Phase Decomposition of the Magnetotelluric Impedance Tensor
}

\author{
Maik Neukirch ${ }^{1}$, now at ${ }^{3}$, Daniel Rudolf ${ }^{2}$, Xavier Garcia ${ }^{1}$ and Savitri Galiana ${ }^{1}$ \\ ${ }^{1}$ Barcelona - Center for Subsurface Imaging, Institut de Ciències del Mar, ICM, CSIC, \\ Passeig Marítim de la Barceloneta, 37, 08003 Barcelona, Spain \\ ${ }^{2}$ Institute for Mathematical Stochastics, Universität Göttingen, Goldschmidtstraße, 7, \\ 37077 Göttingen, Germany \\ ${ }^{3}$ Institut de Ciències de la Terra Jaume Almera, ICTJA, CSIC, Carrer de Lluís Solé $i$ \\ Sabarís, s/n, 08028 Barcelona, Spain
}

(May 31, 2019)

GEO-2018-0352

Running head: MT Amplitude-Phase Decomposition

\begin{abstract}
The introduction of the Phase Tensor marked a breakthrough in the understanding and analysis of electric galvanic distortion effects. It has been used for (distortion free) dimensionality analysis, distortion analysis, mapping and subsurface model inversion. However, the Phase Tensor can only represent half of the information contained in a complete impedance data set. Nevertheless, to avoid uncertainty due to galvanic distortion effects, practitioners often choose to discard half of the measured data and concentrate interpretation efforts on the Phase Tensor part. This work assesses the information loss due to pure Phase Tensor interpretation of a complete impedance data set. To achieve this, a new MT impedance tensor decomposition into the known Phase Tensor and a newly defined
\end{abstract}




\begin{abstract}
Amplitude Tensor is motivated and established. Additionally, the existence and uniqueness of the Amplitude Tensor is proven. Synthetic data is used to illustrate the Amplitude Tensor information content compared to the Phase Tensor. While the Phase Tensor only describes the inductive effects within the subsurface, the Amplitude Tensor holds information about inductive and galvanic effects that can help to identify conductivity or thickness of (conductive) anomalies more accurately than the Phase Tensor. Furthermore, the Amplitude and Phase Tensors sense anomalies at different periods and thus the combination of both provides means to evaluate and differentiate anomaly top depths in the event of data unavailability at extended period ranges, e.g. due to severe noise.
\end{abstract}




\section{INTRODUCTION}

The introduction of the Phase Tensor (Caldwell et al., 2004) marked a breakthrough in the interpretation of Magnetotelluric (MT) impedance data. The Phase Tensor is defined as the matrix multiplication between the inverse real and the imaginary parts of the impedance tensor, $\mathbf{Z}=\mathbf{X}+i \mathbf{Y}$

$$
\mathbf{\Phi}=\mathbf{X}^{-1} \mathbf{Y}
$$

so that for a distorted impedance $\mathbf{Z}_{\mathbf{d}}=\mathbf{D}(\mathbf{X}+i \mathbf{Y})$, the Phase Tensor

$$
\Phi_{\mathbf{d}}=\mathbf{X}^{-1} \mathbf{D}^{-1} \mathbf{D Y}
$$

is free of the distortion $\mathbf{D}$. It contains four algebraic reformulations of the eight components (four real and four imaginary) of the MT impedance tensor such that it is dimensionless (Caldwell et al., 2004). Moreover, the Phase Tensor has been widely accepted and is used for distortion free dimensionality analysis (Martí, 2014), distortion analysis (Bibby et al., 2005), tensor parameter mapping (Booker, 2014) and subsurface model inversion (Patro et al., 2012; Tietze et al., 2015). Still, most tools designed for Phase Tensor data interpretation often discard the second half of the available impedance data in order to maintain independence to the possible presence of galvanic distortion. Furthermore, although the Phase Tensor only indicates changes of subsurface conductivity, the recovery of absolute values by inversion has been demonstrated successfully (Patro et al., 2012; Tietze et al., 2015) if information of several sites overlaps sufficiently and an appropriate a priori model has been chosen. Nevertheless, the authors acknowledge that the neglected amplitude information would be a great asset for Phase Tensor inversions in order to relax these demanding requirements. For a more complete treatment of the Phase Tensor and galvanic distortion the reader is referred to Weidelt and Chave (2012) and Jones (2012). 
In this work we assess what information is omitted if one only chooses to analyse and interpret the Phase Tensor part of a complete impedance data set. Although it is common to interpret Phase Tensor data in combination with other data (e.g. Verticalto-Horizontal magnetic field ratios), we particularly focus on what is neglected from the available impedance data. To achieve this, first, we derive a new algebraic matrix decomposition that explains the full MT impedance tensor in terms of the Phase Tensor and a new Amplitude Tensor. This decomposition can be regarded as a generalisation of the amplitude-phase relation of complex numbers to complex matrices. The Amplitude Tensor is introduced in analogy to the Phase Tensor definition and with a twofold motivation: to obtain an observable that (i) fully represents the impedance together with the Phase Tensor, and (ii) generalises the amplitude-phase representation of scalar complex numbers to matrices with respect to matrix multiplication. We motivate this approach, we prove the existence and uniqueness of the proposed Amplitude Tensor, and we assess, using synthetic data, the information present in the Amplitude Tensor with respect to the Phase Tensor in terms of parameters that are directly related to the geometry of the subsurface conductivity distribution such as the strike angle, dimensionality and anisotropy. The Amplitude Tensor is shown to complement the Phase Tensor quantitatively with respect to the subsurface conductivity distribution. We find that the information contained in the Amplitude Tensor is particularly helpful if the impedance data is sparsely sampled over frequency.

\section{4}




\section{THE AMPLITUDE TENSOR}

\section{Notation and Preliminaries}

For natural numbers $n$ we denote by $\mathbb{C}^{n \times n}$ and $\mathbb{R}^{n \times n}$ the complex and real $n \times n$ matrix spaces, respectively. Let $\mathbb{I}$ be the identity matrix and for $\mathbf{N} \in \mathbb{R}^{n \times n}$ let $\mathbf{N}^{T}$ be the transpose of $\mathbf{N}$. For $\lambda_{1}, \ldots, \lambda_{n} \in \mathbb{R}$ let $\boldsymbol{\Sigma}_{\lambda}$ be a diagonal matrix with $\lambda_{1}, \ldots, \lambda_{n}$ on its diagonal.

Booker (2014) proposes a Phase Tensor parameterization based on geometric considerations, which is closely related to the singular value decomposition (SVD) of the Phase Tensor and decomposes the Phase Tensor in its singular values and two rotations related to the left and right orthogonal SVD matrices. The virtue of this parameterization is that the rotations are related to two undistorted, important inductive observables: the strike angle that is representative for the geologic strike and the skew angle that is associated with subsurface dimensionality. Let us adopt this parameterization for general matrices $\mathbf{X} \in \mathbb{R}^{2 \times 2}$ in the following:

$$
\mathbf{X}=\left(\begin{array}{cc}
X_{1,1} & X_{1,2} \\
X_{2,1} & X_{2,2}
\end{array}\right)=\mathbf{R}\left(-\theta_{X}\right)\left(\begin{array}{cc}
x_{1} & 0 \\
0 & x_{2}
\end{array}\right) \mathbf{R}\left(\psi_{X}\right) \mathbf{R}\left(\theta_{X}\right)=\mathbf{V}_{X} \boldsymbol{\Sigma}_{X} \mathbf{W}_{X}^{T}
$$

where $\theta_{X}=\arccos \left(\operatorname{Tr}\left(\mathbf{V}_{X}\right) / 2\right)$ is the angle between the cartesian and the matrix ellipse coordinates that, for the Phase Tensor, represents the geologic strike angle in $2 \mathrm{D}$ cases (Booker, 2014);

$$
\psi_{X}= \begin{cases}\arctan \frac{X_{1,2}-X_{2,1}}{X_{1,1}+X_{2,2}}, & \text { if } 0 \leq\left|X_{1,2}-X_{2,1}\right| \leq\left|X_{1,1}+X_{2,2}\right| \neq 0, \\ \operatorname{arccot} \frac{X_{1,1}+X_{2,2}}{X_{1,2}-X_{2,1}}, & \text { if }\left|X_{1,2}-X_{2,1}\right|>\left|X_{1,1}+X_{2,2}\right| \text { and } \frac{X_{1,1}+X_{2,2}}{X_{1,2}-X_{2,1}} \geq 0, \\ \operatorname{arccot} \frac{X_{1,1}+X_{2,2}}{X_{1,2}-X_{2,1}}-\pi, & \text { if }\left|X_{1,2}-X_{2,1}\right|>\left|X_{1,1}+X_{2,2}\right| \text { and } \frac{X_{1,1}+X_{2,2}}{X_{1,2}-X_{2,1}}<0,\end{cases}
$$

is the normalised matrix skew angle (Booker, 2014); $x_{1}$ and $x_{2}$, in the diagonal matrix $\boldsymbol{\Sigma}_{X}$, are the singular values of $\mathbf{X}$; and $\mathbf{V}_{X}=\mathbf{R}\left(-\theta_{X}\right)$ and $\mathbf{W}_{X}=\mathbf{R}\left(-\psi_{X}-\theta_{X}\right)$ are the left and 
right orthogonal SVD matrices, with the rotation by $\delta$ defined as:

$$
\mathbf{R}(\delta)=\left(\begin{array}{cc}
\cos \delta & \sin \delta \\
-\sin \delta & \cos \delta
\end{array}\right) .
$$

The normalised matrix skew angle in (4) ensures that the definition of $\hat{\psi}_{X}=\arctan \frac{X_{1,2}-X_{2,1}}{X_{1,1}+X_{2,2}}$ by Booker (2014) is defined for diagonal and anti-diagonal matrices $\mathbf{X}$ by expressing the limit of the arctangent function with arccotangent, viz. $\lim _{y \rightarrow \pm 0} \arctan \frac{x}{y}= \pm \frac{\pi}{2}=\operatorname{arccot} \frac{y}{x}-$ $\frac{\pi}{2} \pm \frac{\pi}{2}, \forall x>0$, and therewith, provides improved numerical stability for $\psi_{X}>45^{\circ}$.

\section{Amplitude-Phase Decomposition}

We introduce the Amplitude-Phase decomposition as a new algebraic decomposition of a complex matrix $\mathbf{M} \in \mathbb{C}^{n \times n}$ into real-valued Amplitude and Phase matrices, $\mathbf{P} \in \mathbb{R}^{n \times n}$ and $\Phi \in \mathbb{R}^{n \times n}$, respectively. We prove the existence of such a decomposition and argue for the uniqueness of a principal solution in the appendix. We derive this formulation in analogy to the polar form of complex numbers

$$
z=\varrho \exp (i \varphi)=\varrho(\cos \varphi+i \sin \varphi)
$$

using the property that the phase, $\varphi$, and the amplitude, $\varrho$, are real-valued, and that the Pythagorean identity:

$$
\cos ^{2} \varphi+\sin ^{2} \varphi=1
$$

is satisfied.

Thus, let us represent $\mathbf{M} \in \mathbb{C}^{n \times n}$ in terms of two real-valued matrices, $\mathbf{P}$ (read capital greek letter Rho) and $\boldsymbol{\Phi}$, associated with the amplitude $(\varrho)$ and phase $(\varphi)$ of the polar form in (6), with defined functions $c(\boldsymbol{\Phi}), s(\mathbf{\Phi})$ and $e(\boldsymbol{\Phi})$ corresponding to $\cos \varphi, \sin \varphi$ and 
$\exp (i \varphi)$

$$
\mathbf{M}=\mathbf{P} e(\boldsymbol{\Phi})=\mathbf{P}(c(\boldsymbol{\Phi})+i s(\boldsymbol{\Phi}))
$$

In particular, the sum of squares of the amplitude independent terms shall be unity in analogy to the Pythagorean identity:

$$
c(\boldsymbol{\Phi}) c(\boldsymbol{\Phi})^{T}+s(\boldsymbol{\Phi}) s(\boldsymbol{\Phi})^{T}=\mathbb{I} .
$$

The definition of $\boldsymbol{\Phi}$ in (1), the decomposition in (8) and the constraint in (9) yield solutions for $e$ such that a real-valued amplitude matrix $\mathbf{P}$ always exists if $e(\boldsymbol{\Phi})$ is invertible. Further, it can be argued that one fundamental solution for $\mathbf{P}$ exists where $e(\mathbf{\Phi})$ shares SVD properties with the unique phase matrix (see appendix).

\section{Definition of the Amplitude Tensor}

Let us represent the invertible, complex-valued $2 \times 2 \mathrm{MT}$ impedance tensor, $\mathbf{Z}=\mathbf{X}+i \mathbf{Y}$, using (8) and constraint by (9) as a multiplication of a real-valued Amplitude Tensor, $\mathbf{P}$, with a function, $e$, of the invertible, real-valued Phase Tensor, $\mathbf{\Phi}=\mathbf{X}^{-1} \mathbf{Y}$. Then, we obtain the following fundamental solutions (see appendix) for $c(\mathbf{\Phi}), s(\mathbf{\Phi})$ and $e(\mathbf{\Phi})$ :

$$
c(\boldsymbol{\Phi})=\left(\mathbb{I}+\mathbf{\Phi} \boldsymbol{\Phi}^{T}\right)^{-\frac{1}{2}}, \quad s(\boldsymbol{\Phi})=\left(\mathbb{I}+\mathbf{\Phi} \boldsymbol{\Phi}^{T}\right)^{-\frac{1}{2}} \boldsymbol{\Phi}, \quad e(\boldsymbol{\Phi})=c(\boldsymbol{\Phi})+i s(\boldsymbol{\Phi}),
$$

where the superscript $-\frac{1}{2}$ denotes the matrix inverse of the matrix square root. Hence, as simply follows from (8), a unique and real-valued Amplitude Tensor can be defined as:

$$
\mathbf{P}=\mathbf{Z}(e(\boldsymbol{\Phi}))^{-1}
$$




\section{Discussion on Alternatives to the Amplitude Tensor Approach}

It could be argued that either real or imaginary part of the impedance, viz. $\mathbf{Z}=\mathbf{X}(\mathbb{I}+i \boldsymbol{\Phi})$ or $\mathbf{Z}=\mathbf{Y}\left(\boldsymbol{\Phi}^{-1}+i \mathbb{I}\right)$, can complement the MT Phase Tensor equally well as the proposed Amplitude Tensor. If this is true, the advantage would be that either of them is readily available without further computation while they appear to offer similar decomposition and interpretation capabilities as the proposed Amplitude Tensor. Thus, why do we propose an Amplitude Tensor? While both, real and imaginary impedances, contain the impedance amplitude, both of them also contain Phase Tensor information. This is immediately clear for the special case of the Amplitude-Phase decomposition for scalar complex numbers, i.e. for a $1 \mathrm{D}$ MT scenario where $z=\Re z+i \Im z=\rho e^{i \phi}=\rho \cos \phi+i \rho \sin \phi$, but it also holds for the decomposition of matrices. In order to find the analogue for matrices, we represent the Amplitude-Phase decomposition (8) of the impedance tensor with SVDs of the Amplitude and Phase Tensors (see explicit SVD of Phase Tensor functions in the appendix, i.e. (A-4) and (A-5)) according to (3):

$$
\begin{aligned}
\mathbf{Z} & =\mathbf{X}+i \mathbf{Y}=\mathbf{P} e(\boldsymbol{\Phi})=\mathbf{P}(c(\boldsymbol{\Phi})+i s(\boldsymbol{\Phi})) \\
& =\mathbf{P}(\mathbf{C}+i \mathbf{S}) \\
& =\left(\mathbf{V}_{P} \boldsymbol{\Sigma}_{P} \mathbf{W}_{P}^{T}\right)\left(\left(\mathbf{V}_{\Phi} \boldsymbol{\Sigma}_{c} \mathbf{V}_{\Phi}^{T}\right)+i\left(\mathbf{V}_{\Phi} \boldsymbol{\Sigma}_{s} \mathbf{W}_{\Phi}^{T}\right)\right) \\
& =\left(\mathbf{R}\left(-\theta_{P}\right) \boldsymbol{\Sigma}_{P} \mathbf{R}\left(\psi_{P}\right) \mathbf{R}\left(\theta_{P}\right)\right)\left(\mathbf{R}\left(-\theta_{\Phi}\right)\left(\boldsymbol{\Sigma}_{c}+i \boldsymbol{\Sigma}_{s} \mathbf{R}\left(\psi_{\Phi}\right)\right) \mathbf{R}\left(\theta_{\Phi}\right)\right)
\end{aligned}
$$

This SVD expansion of the Amplitude-Phase decomposition reveals that real and imaginary impedances can be formulated as mixtures of the Amplitude and Phase Tensor parameters:

$$
\begin{aligned}
& \mathbf{X}(\mathbf{P}, \boldsymbol{\Phi})=\mathbf{R}\left(-\theta_{P}\right) \boldsymbol{\Sigma}_{P} \mathbf{R}\left(\psi_{P}\right) \mathbf{R}\left(\theta_{P}\right) \mathbf{R}\left(-\theta_{\Phi}\right) \boldsymbol{\Sigma}_{c} \mathbf{R}\left(\theta_{\Phi}\right) \quad \text { and } \\
& \mathbf{Y}(\mathbf{P}, \boldsymbol{\Phi})=\mathbf{R}\left(-\theta_{P}\right) \boldsymbol{\Sigma}_{P} \mathbf{R}\left(\psi_{P}\right) \mathbf{R}\left(\theta_{P}\right) \mathbf{R}\left(-\theta_{\Phi}\right) \boldsymbol{\Sigma}_{s} \mathbf{R}\left(\psi_{\Phi}\right) \mathbf{R}\left(\theta_{\Phi}\right) .
\end{aligned}
$$


In contrast, and by the definition of the Amplitude Phase Decomposition, the Phase Tensor parameters cannot be related to the Amplitude Tensor parameters, viz.:

$$
\mathbf{\Phi}=\mathbf{X}^{-1} \mathbf{Y}=(\mathbf{P C})^{-1} \mathbf{P S}=\mathbf{C}^{-1} \mathbf{P}^{-1} \mathbf{P S}=\mathbf{C}^{-1} \mathbf{S}
$$

Of course, $\mathbf{C}=\mathbf{P}^{-1} \mathbf{X}$ and $\mathbf{S}=\mathbf{P}^{-1} \mathbf{Y}$ themselves remain functions of the Amplitude Tensor and the real and imaginary parts of the impedance, respectively, but (15) is formulated in such a way that the contribution of the Amplitude Tensor cancels out and thus leaves no trace in the Phase Tensor. In fact, this is a major motivation for the definition of the Amplitude Tensor in the Amplitude Phase Decomposition: to quantify the impedance information that is lost due to the definition of the Phase Tensor.

Moreover, from (13) and (14), we find that any interpretation of real or imaginary impedance tensor parameters will be convoluted with Phase Tensor information and therefore, impede any joint interpretation between either real or imaginary impedance and the Phase Tensor due to inherent redundancy in the contained information. Note that this conclusion applies naturally to all impedance derivatives which also contain Phase Tensor information, such as the Apparent Resistivity Tensor (ART) introduced by Weckmann et al. (2003). While some impedance derivatives are a better choice than others to complement the Phase Tensor, i.e. the ART may be better suited than the real and imaginary parts as we will show later, only the Amplitude Tensor is explicitly defined to cancel out in the Phase Tensor definition and thus represents exclusively the lost information in the Phase Tensor with respect to the impedance.

Figure 1 should be about here or on the following page.

In Figure 1, we compare the decomposition parameters for the Phase, Amplitude, Apparent Resistivity Tensors, and real and imaginary part of the impedance. The model 
corresponding to these data was taken from Caldwell et al. (2004) and will be discussed in more detail later in the Examples section. The alternative representations to the Amplitude Tensor, i.e. the ART, do have merit for the visualisation purposes for which they have been designed for. However, a joint interpretation of these and the Phase Tensor must be performed with great care, since part of the presented information overlaps and it is not a simple task to differentiate which information might be complementary and which is redundant between the tensors. We argue that the Amplitude and Phase Tensors form a complementary pair and therefore, the proposed Amplitude Tensor is a better choice than either real or imaginary impedance for a decomposition of the impedance tensor based on the Phase Tensor. We do appreciate, however, that especially the singular values of the ART and the Amplitude Tensor agree almost entirely (see Figure 1), while both, the real and imaginary parts consistently over- and underestimate the present apparent resistivity. There are, nevertheless, significant differences in the skew and strike angles between the Amplitude Tensor and the ART. The ART strongly overestimates the skew angle, while the Amplitude Tensor skew is close to the skew of real and imaginary part, which represent the impedance skew and dimensionality. The large skew of the ART can be attributed to its definition in which the impedance components are mixed non-linearly and therefore it does not necessarily reflect subsurface dimensionality correctly. The Amplitude Tensor is defined such that it contains the impedance information that is lost due to the Phase Tensor definition and therefore, all deviations of its geometric parameters to the Phase Tensor parameters offer new, untapped information with respect to the Phase Tensor. On the other hand, the ART cannot be guaranteed to be free of any information already contained in the Phase Tensor and interpretations based on discrepancies between its parameters and the Phase Tensor may be affected by a varying degree of redundancy that is near impossible to 
gauge in any practical way.

\section{Dimensionality}

In the $1 \mathrm{D}$ case, the MT Impedance, the Phase and the Amplitude Tensors simplify to:

$$
\mathbf{Z}_{D}=\left(\begin{array}{cc}
0 & z_{1,2} \\
-z_{2,1} & 0
\end{array}\right), \quad \boldsymbol{\Phi}_{D}=\left(\begin{array}{cc}
\phi_{1,1} & 0 \\
0 & \phi_{2,2}
\end{array}\right), \quad \text { and } \quad \mathbf{P}_{D}=\left(\begin{array}{cc}
0 & \rho_{1,2} \\
-\rho_{2,1} & 0
\end{array}\right),
$$

with $z_{1,2}=z_{2,1}=z_{1 D}, \phi_{1,1}=\phi_{2,2}=\phi_{1 D}$ and $\rho_{1,2}=\rho_{2,1}=\rho_{1 D}$. Considering the $1 D$ impedance given by $z_{1 D}=\left|z_{1 D}\right| \exp \left(i \arctan \phi_{1 D}\right)$, where $\rho_{1 D}=\left|z_{1 D}\right|$, the Amplitude Tensor can be expressed with the apparent resistivity by $\rho_{1 D}=\sqrt{\omega \mu_{0} \rho_{1 D}^{\text {app }}}$, with angular frequency $\omega=2 \pi / T$, period $T$ and magnetic permeability of free space $\mu_{0}$. From equations (4) and (16), we have that the skew angle $\psi_{P}=\pi / 2$ at all frequencies. This corresponds to $\psi_{\Phi}=0$, because the $1 \mathrm{D}$ Amplitude Tensor is anti-diagonal like the impedance. Then, for simplicity, we define the normalized amplitude skew angle as:

$$
\psi_{P}^{\text {norm }}=\frac{\pi}{2}-\psi_{P}
$$

so that it equals zero for $1 \mathrm{D}$ situations in analogy to the phase skew angle. In other words, the Amplitude Tensor can be represented by a unity matrix rotated by $\psi_{P}=\frac{\pi}{2}$ and multiplied by the scalar $\left|z_{1 D}\right|$ and therefore, the singular values $\rho_{1}$ and $\rho_{2}$ are equal.

In the 2D case, the MT impedance, Phase and Amplitude Tensors simplify to (16), with $z_{1,2}=z_{T E}, z_{2,1}=z_{T M}, \phi_{1,1}=\phi_{T E}, \phi_{2,2}=\phi_{T M}, \rho_{1,2}=\rho_{T E}$ and $\rho_{2,1}=\rho_{T M}$, in the transverse electric (TE) and transverse magnetic (TM) modes. The 2D impedances are given by $z_{m}=\left|z_{m}\right| \exp \left(i \arctan \phi_{m}\right)$, with $\rho_{m}=\left|z_{m}\right|$ and $m$ denoting either the TE or TM mode, the Amplitude Tensor can be associated with the apparent resistivity, $\rho_{m}^{\text {app }}$, through $\rho_{m}=\sqrt{\omega \mu_{0} \rho_{m}^{\mathrm{app}}}$. As for the $1 \mathrm{D}$ case, it can be seen from (4) that the skew angle 
is $\psi_{P}=\frac{\pi}{2}$. In addition the strike angle parameters of the Amplitude and Phase Tensors must be equal in this case, since an arbitrary oriented $2 \mathrm{D}$ impedance, $\mathbf{Z}_{2 \mathrm{D} \text {,rot }}$, can always be expressed as a product of its local $2 \mathrm{D}$ form, $\mathbf{Z}_{2 \mathrm{D}}$, and a coordinate rotation by the strike angle $\theta_{z}: \mathbf{Z}_{2 \mathrm{D}, \text { rot }}=\mathbf{R}\left(\theta_{z}\right) \mathbf{Z}_{2 \mathrm{D}} \mathbf{R}\left(-\theta_{z}\right)$. Then, identifying the phase strike angle $\theta_{\Phi}$ with $\theta_{z}$ and comparing the resulting, diagonal and local $2 \mathrm{D}$ form $\mathbf{Z}_{2 \mathrm{D}}$ with (12):

$$
\mathbf{Z}_{2 \mathrm{D}}=\mathbf{R}\left(-\left(\theta_{P}-\theta_{\Phi}\right)\right) \boldsymbol{\Sigma}_{P} \mathbf{R}(0.5 \pi) \mathbf{R}\left(\theta_{P}-\theta_{\Phi}\right)\left(\boldsymbol{\Sigma}_{c}+i \boldsymbol{\Sigma}_{s}\right)
$$

which implies that the strike angle difference between the Amplitude and Phase Tensors is $\theta_{P}-\theta_{\Phi}=0$ for $2 \mathrm{D}$ media due to the required diagonal form of $\mathbf{Z}_{2 \mathrm{D}}$. Further, the singular values of Phase and Amplitude Tensors in (16) equal the impedance phase and amplitude values, respectively, of the TE and TM modes, similarly to the $1 \mathrm{D}$ case.

Lastly, the change over period of all Amplitude Tensor parameters is the signature of a 3D subsurface. Note that in 3D situations the singular values of the Amplitude Tensor can also be defined as apparent resistivity but interpreting them in terms of subsurface structure is not as straight forward as for $1 \mathrm{D}$ or $2 \mathrm{D}$ cases because, then, their interpretation must include the amplitude skew and strike angles.

\section{EXAMPLES}

We analyse the information content of the Amplitude Tensor with respect to the Phase Tensor by mapping the tensor parameters and discuss the information that can be extracted from each. Thereby we focus on identifying which information is neglected if only the Phase Tensor is interpreted.

We study data sets generated for two different synthetic models taken from the literature. The parameter maps for each of these data sets are given in Figures 2 to 5 . Each plot 
contains all four parameters (mean singular values, relative anisotropy, strike and skew angles) of the corresponding tensor. The mean singular values $\ln \rho$ and $\phi$, as defined in the appendix by (B-2) and (B-3), are color-coded in degrees (Phase Tensor) and in the natural logarithm of the apparent resistivity values (Amplitude Tensor). The relative anisotropy parameters, $\rho_{a}$ and $\phi_{a}$, as defined in the appendix by (B-1), are plotted as an ellipse with major axis $1+\rho_{a}$ and $1+\phi_{a}$ and minor axis $1-\rho_{a}$ and $1-\phi_{a}$, respectively. The unit circles of $\rho_{a}=2 / 3, \rho_{a}=0$ and $\rho_{a}=-2 / 3$, and similarly for $\phi_{a}$, are provided for comparison at the bottom right corner of the plots. The strike angle is typically aligned to the major or minor axis of the ellipse but additionally highlighted by a black line. Finally, the skew angle is displayed qualitatively as white $\left(0^{\circ}\right)$, grey $\left(\leq 6^{\circ}\right)$ and black $\left(>6^{\circ}\right)$ unit circle to illustrate regimes of 2D, quasi 2D (according to Booker, 2014) and 3D, respectively.

\section{Model of Caldwell et al. (2004)}

Figure 2 should be within this subsection.

The first model we study was proposed by Caldwell et al. (2004) to introduce the Phase Tensor and it demonstrates the significance of spatial strike angle variation with respect to data three-dimensionality. We study this model to identify additional information that can be extracted from the Amplitude Tensor.

The model consists of a homogeneous background of $100 \Omega \mathrm{m}$ with a $10 \Omega \mathrm{m}$ cube to the West, with sides of $250 \mathrm{~m}$, with its top $50 \mathrm{~m}$ below the surface (only half covered by the map), and a much larger rectangular $\left(7 \times 3 \times 3 \mathrm{~km}^{3}\right) 1 \Omega \mathrm{m}$ conductive body to the SouthEast, buried with its top $1.5 \mathrm{~km}$ below the surface (only a small portion covered by the map). Figure 2 shows map views of the Phase and Amplitude Tensors at various periods 
for this model and outlines the underlying model structure for easy reference.

The Phase Tensor begins to sense the small and shallow Western body at around $10^{-4} \mathrm{~S}$ (see Supplements) and the Amplitude Tensor at around $6.8 \cdot 10^{-4} \mathrm{~s}$ as can be seen by the reduced mean of the logarithmic apparent resistivity singular values at the position of the anomaly compared to the rest of the modelled data.

At around a period of $4.6 \cdot 10^{-3} \mathrm{~s}$ we can observe that the Phase Tensor has the widest extend of sensitivity to the small Western body indicated by the area with significant strike angles. The data where the mean of the phase singular values are $\phi>45^{\circ}$ clearly indicate the position and the conductive nature of the body. Outside the position of the conductive body, the anisotropy ellipses and strike angles are both elongated and measurable, but they are not in the area below the conductive body and therewith this difference specifies also the position and shape of the body. With increasing period the Phase Tensor parameter values approach their homogenous half-space values $\left(45^{\circ}\right.$ phase scale, zero anisotropy, zero skew angle and indeterminable strike angle) until the Western body is indeterminable at a period of $0.1 \mathrm{~s}$. Then, at a period around $0.22 \mathrm{~s}$ (see Supplements), the increase of the anisotropy values and the appearance of a measurable strike angle indicate that the Phase Tensor begins to sense the deep and large South-Eastern anomaly. At a period around 1s, the area in which the large anomaly is detected by the Phase Tensor parameters is largest and thereafter the area of influence decreases again until the response is below measurable at $32 \mathrm{~s}$. From these observations, we can conclude that the Phase Tensor is sensitive only to structures of specific sizes and depth at a given period, i.e. only for short periods small, near-surface bodies affect the Phase Tensor parameters and at longer periods deeper and larger structures are sensed.

14 
On the other hand, the Amplitude Tensor behaves quite differently to the Phase Tensor. At low periods, i.e. at $6.8 \cdot 10^{-4} \mathrm{~s}$ and $4.6 \cdot 10^{-3} \mathrm{~s}$, the averages of the Amplitude Tensor singular values at the West of the model are below the background resistivity of $100 \Omega m$ indicating the position and conductive nature of the western anomaly. Additionally, at the period of $4.6 \cdot 10^{-3} \mathrm{~s}$, the amplitude strike and anisotropy values are specifying the Western body's shape and position just as we observed for the Phase Tensor. The notable difference towards the Phase Tensor data is that the amplitude begins to sense the shallow anomaly at longer periods and that the effect of the anomaly on the Amplitude Tensor does not diminish even for the longest periods. Observing the Amplitude Tensor parameters at a larger period, i.e. at $0.1 \mathrm{~s}$ and $1 \mathrm{~s}$, the parameters show the same behavior as for lower period values, indicating that the Amplitude Tensor is still affected by the near surface anomaly in contrast to what we observe for the Phase Tensor. At these larger periods, we also observe that the apparent resistivity, represented by the mean of the amplitude singular values, decreases over the shallower body's position approaching values around $10 \Omega m$ and that the anisotropy and strike angle parameters begin to be affected by the large South-Eastern anomaly.

At the longest period of $32 \mathrm{~s}$ (and longer periods not shown here, see Supplements), the amplitude scale parameter decreases towards the South-East indicating the presence of the large and deep conductive body, and the anisotropy and strike angle parameters (considering the $90^{\circ}$ strike angle ambiguity) point towards its position. At this period, the small and shallow Western and the large and deep South-Eastern bodies can still be observed in the amplitude parameters but not in the phase parameters. The absence of influence from the anomalous bodies in the phase parameters and its presence in the amplitude parameters indicates that the effects observed by the presence of such bodies are not governed by 
inductive regime at this period but by a purely galvanic one.

\section{D MT Modeling and Inversion Workshop 2016 - Secret Model \#3}

The model used in the previous example, while insightful, it lacks illustration of the skew angle parameter and it exhibits a low model complexity. Thus, we analyze an additional synthetic model that was particularly designed to contain 3D data and to emulate more closely a complex subsurface. We take it from the 3D MT Modeling and Inversion Workshop celebrated in 2016. Additionally, we note that we have no knowledge about the underlying (yet unpublished) model.

The secret model \#3 is a large scale model that represents an ocean-continent setting inspired by the North American west coast, which includes bathymetry and topography and is noted as showing strong ocean effects. Therewith it contains more complex and more realistic data than the previous example. Figures 3,4 and 5 display parameter map plots at a selection of periods from this model. The specifics of the data can be found on the website ( http://www.dias.ie/mt3dinv3/Home.html ) but model specifics have not yet been published.

Figure 3 should be around here.

In Figure 3, we plot the Phase and Amplitude Tensor parameters at the period of $0.4 \mathrm{~s}$. It can be observed that, at this period and at each station, the parameters of both tensors are almost all identical, with phase and amplitude mean singular values of $45^{\circ}$ and $100 \Omega m$, respectively, with indeterminable strike angle, zero anisotropy and zero skew angle, indicating that they both sense a homogeneous subsurface. At a period of $4 \mathrm{~s}$, still in Figure 3 , the averages of the phase singular values shows different shallow conductive anomalies $(E$ : 
and $F$ : K18 to N18), which are not yet obvious in the apparent resistivity associated to the mean logarithmic amplitude singular values, with a constant value of $100 \Omega m$. Only for the anomaly $A$, the amplitude apparent resistivity is around $50 \Omega m$, therefore, the conductivity anomaly in this region is likely shallower than the others. At the same period, we observe very large phase anisotropy values and large phase strike angle deviations which must be due to extreme coastal effects. However, it is not yet possible to clearly appreciate these effects in the amplitude parameters. Note that here the amplitude parameters provide a means to differentiate information from the phase data. The amplitude data indicates that anomaly $A$ is shallower than the other discovered anomalies in the phase data. While the information about the relative depths of the anomalies must be also present in the Phase Tensor data and, in fact, we can observe anomaly $A$ in the phase data at the period below $4 \mathrm{~s}$ (see Supplements), such a fine period sampling is not always available with real impedance data where period bands may be masked due to noise. This observation shows that the amplitude data can help to mitigate information loss due to coarse period sampling or the presence of noise.

Figure 4 should be around here.

In Figure 4, at a period of 40 s, the Amplitude Tensor shows unmistakably the severe coastal effect represented by large anisotropy values, and the different shallow anomalies represented by large phase anisotropy values, which could already be appreciated in the Phase Tensor parameters at $4 \mathrm{~s}$. At periods between $40 \mathrm{~s}$ and $400 \mathrm{~s}$, the large values of the phase and amplitude anisotropy parameters that we associate to the coastal effect continue to increase and additionally, in the region next to the aforementioned anomaly $C$, the averages of the amplitude (phase) singular values decrease (increase), showing the presence 
of a new, larger and deeper conductive body to the North ( $G$ : A17-21 to E17-21). At $400 \mathrm{~s}$, the described response of the mean of the phase singular values to the shallow conductive structure cannot be appreciated anymore, but their effects are still present in the mean of the amplitude singular values indicating that the electromagnetic processes in these anomalies at this period are governed by galvanic effects. However, at the same period, the mean of the phase singular values at anomaly $F$ has decreased below $45^{\circ}$ indicating the presence of a resistive body just in a position beneath the previously detected conductive anomaly $A$.

\section{Figure 5 should be around here.}

In Figure 5, at 4,000 s, the large values of the anisotropy parameter associated to the coastal effect diminish for the Phase (reduced sensitivity) and reduces for the Amplitude (less dominance) Tensors. Moreover, at this period and also at 40,000 s, the Phase Tensor strike angle is aligned to the coastline (note the $90^{\circ}$ ambiguity) which indicates that the underlying bedrock is either $1 \mathrm{D}$ anisotropic or features a $2 \mathrm{D}$ conductivity distribution but without any clear information on its structure. On the other hand, at 4,000 s and 40,000s, the mean values of the Amplitude Tensor singular values detect two distinct areas, in one area the associated apparent resistivity values lie between $10 \Omega m$ and $20 \Omega m$ and in the other they are between $60 \Omega m$ and $100 \Omega m$, clearly distinguishing, in contrast to the phase data, the resistive continental and the conductive marine regions of the model. Further, at these periods, the Amplitude Tensor still shows the dominant features of the model indicating strong galvanic effects governing the electromagnetic processes in the anomalies $B, C, F$ and $G$. These anomalies are mainly conductive as indicated by the apparent resistivity values. We first detected them at the period of $40 \mathrm{~s}$ and therefore they are shallower than the skin depth at a period of 40,000s. The dominant character of these conductive anomalies in the Amplitude Tensor data could be associated to relatively high conductivity or due to a 
particularly large thickness, both of these interpretations could not be made from Phase Tensor data alone.

\section{CONCLUSIONS}

The Phase Tensor can only represent half of the information contained in a complete impedance data set. Nevertheless, to avoid uncertainty due to galvanic distortion effects, practitioners often choose to discard half of the measured data and concentrate interpretation efforts on the Phase Tensor part of the data. In this work, we discuss the information loss due to pure Phase Tensor interpretation of complete impedance data sets. We propose and motivate a new MT impedance tensor decomposition into the known Phase Tensor and a newly defined Amplitude Tensor. The purpose of the decomposition is to provide a mean to separate Phase Tensor information and what is left out by the Phase Tensor, i.e. what is lost when only using the Phase Tensor, from the MT impedance tensor. We show that the Amplitude Tensor is complementary to the Phase Tensor and both together form a complete decomposition of the impedance tensor. We proof existence and uniqueness of the Amplitude Tensor, discuss its properties related to subsurface dimensionality and illustrate its information content on synthetic data compared to the information within the Phase Tensor.

Further, we compare the Amplitude Tensor parameters to alternative representations: the Apparent Resistivity Tensor and the real and imaginary parts of the impedance tensor. The Apparent Resistivity Tensor indicates accurately the apparent resistivity of the subsurface but it cannot accurately reproduce impedance dimensionality. On the other hand, the real and imaginary parts provide accurate means to estimate the impedance dimensionality but neither yields the correct apparent resistivity. The Amplitude Tensor is shown to offer 
nearly the same insights as the Apparent Resistivity Tensor with respect to the quantitative assessment of subsurface resistivity (determinant and anisotropy) and it contains very similar dimensionality information (strike and skew) as the real and imaginary impedances. Thus, the Amplitude Tensor combines the desirable information of the Apparent Resistivity Tensor and the impedance parts, rendering it the most ideal analysis complement to the Phase Tensor.

We conclude that the Amplitude Tensor contains distinct information with respect to the Phase Tensor. While the Phase Tensor only describes the inductive effects within the subsurface, the Amplitude Tensor holds information about inductive and galvanic effects. In the examples presented, we noticed that the Amplitude and Phase Tensors sense anomalies at different periods and thus the combination of both provides means to qualitatively evaluate and differentiate anomaly depths in the event of data unavailability at extended period ranges. Furthermore, the galvanic effects stored in the Amplitude Tensor can help to identify conductivity or thickness of (conductive) anomalies more accurately than uniquely using the Phase Tensor. The Amplitude Tensor presented in this work is complementary to the Phase Tensor and it contains integrated information about inductive and galvanic effects of the subsurface. Therewith, it could also be used as an additional source of independent information for Phase Tensor inversions, because it adds galvanic to the, otherwise, purely inductive constraints to the model.

\section{ACKNOWLEDGMENTS}

This work was funded by Repsol under the framework of the CODOS-2 project. The MT3D workshop dataset is publicly available at https://www.dias.ie/mt3dinv3/Home.html for download and further details. For this we especially thank the workshop organisers and 
the model creators, Alan Jones, Marion Miensopust, Pilar Queralt, Agata Siniscalchi and Jan Vozar. The constructive and smart criticism of Anna Avdeeva, John Booker, Gary Egbert, Colin Farquharson, Alan Jones, Ute Weckmann, and three anonymous reviewers have helped us to improve earlier versions of this paper.

\section{SUPPLEMENTS}

A MatLab executable to compute and plot Amplitude and Phase Tensor parameters is available at:

https://www.dropbox.com/sh/61g7vbl2z8dtpey/AACmbGBsbpTGzW9nrdrc1u37a?dl=0

for academic use. At the same location, parameter maps of the models discussed in this work are available for a finer and longer period range for the interested reader as well as the data files for the first example.

\section{APPENDIX A}

\section{PROOFS FOR THE AMPLITUDE-PHASE DECOMPOSITION}

Let $\mathbf{M} \in \mathbb{C}^{n \times n}$ with $\mathbf{M}=\mathbf{X}+i \mathbf{Y}$, where $\mathbf{X}, \mathbf{Y} \in \mathbb{R}^{n \times n}$ are invertible. Set $\mathbf{\Phi}=\mathbf{X}^{-1} \mathbf{Y}$ and:

$$
\begin{aligned}
& \mathbf{C}_{0}=\left(\mathbb{I}+\boldsymbol{\Phi} \boldsymbol{\Phi}^{T}\right)^{-1 / 2}, \\
& \mathbf{S}_{0}=\mathbf{C}_{0} \boldsymbol{\Phi} \\
& \mathbf{P}_{0}=\mathbf{X C}_{0}^{-1}=\mathbf{Y S}_{0}^{-1},
\end{aligned}
$$

where $\mathbb{I} \in \mathbb{R}^{n \times n}$ denotes the identity matrix. 
Theorem 1. For any orthogonal $\mathbf{U} \in \mathbb{R}^{n \times n}$ the matrices,

$$
\mathbf{C}=\mathbf{U C}_{0}, \quad \mathbf{S}=\mathbf{U S}_{0}, \quad \mathbf{P}=\mathbf{P}_{0} \mathbf{U}^{T}
$$

satisfy:

$$
\begin{aligned}
\mathbf{S} & =\mathbf{C} \boldsymbol{\Phi}, \\
\mathbf{M} & =\mathbf{P}(\mathbf{C}+i \mathbf{S}), \\
\mathbb{I} & =\mathbf{C C}^{T}+\mathbf{S S}^{T} .
\end{aligned}
$$

Proof. By $\mathbf{S}_{0}=\mathbf{C}_{0} \mathbf{\Phi}$ we obviously have (A-1). Equation (A-3) follows from $\mathbf{C}_{0}=\mathbf{C}_{0}^{T}$, $\mathbf{U U}^{T}=\mathbb{I}$ and

$$
\mathbf{C C}^{T}+\mathbf{S S}^{T}=\mathbf{U} \mathbf{C}_{0}\left(\mathbb{I}+\mathbf{\Phi} \boldsymbol{\Phi}^{T}\right) \mathbf{C}_{0}^{T} \mathbf{U}^{T}=\mathbb{I}
$$

Since $\mathbf{U}^{T} \mathbf{U}=\mathbb{I}$ we obtain (A-2) by:

$$
\mathbf{P}(\mathbf{C}+i \mathbf{S})=\mathbf{P}_{0} \mathbf{U}^{T} \mathbf{U C}_{0}(\mathbb{I}+i \boldsymbol{\Phi})=\mathbf{X}\left(\mathbb{I}+i \mathbf{X}^{-1} \mathbf{Y}\right)=\mathbf{M}
$$

Theorem 2. For matrices $\mathbf{C}, \mathbf{S}, \mathbf{P} \in \mathbb{R}^{n \times n}$ which satisfy (A-1), (A-2) and (A-3), there exists an orthogonal matrix $\mathbf{U} \in \mathbb{R}^{n \times n}$ so that:

$$
\mathbf{C}=\mathbf{U C}_{0}, \quad \mathbf{S}=\mathbf{U S}_{0}, \quad \mathbf{P}=\mathbf{P}_{0} \mathbf{U}^{T}
$$

Proof. We set $\mathbf{U}=\mathbf{C C}_{0}^{-1}$ and verify that $\mathbf{U}$ is an orthogonal matrix with $\mathbf{C}=\mathbf{U C}_{0}$, $\mathbf{S}=\mathbf{U S}_{0}$ and $\mathbf{P}=\mathbf{P}_{0} \mathbf{U}^{T}$. We have by $\mathbf{C}_{0}^{-1}=\left(\mathbf{C}_{0}^{-1}\right)^{T},(\mathrm{~A}-1)$ and (A-3) the orthogonality:

$$
\mathbf{U} \mathbf{U}^{T}=\mathbf{C C}_{0}^{-1} \mathbf{C}_{0}^{-1} \mathbf{C}^{T}=\mathbf{C}\left(\mathbb{I}+\boldsymbol{\Phi} \boldsymbol{\Phi}^{T}\right) \mathbf{C}^{T}=\mathbf{C C}^{T}+\mathbf{S S}^{T}=\mathbb{I}
$$

Then, clearly $\mathbf{C}=\mathbf{U C}_{0}$ and by (A-1) holds:

$$
\mathbf{U S}_{0}=\mathbf{C C}_{0}^{-1} \mathbf{S}_{0}=\mathbf{C \Phi}=\mathbf{S}
$$


Finally, from $(\mathrm{A}-2)$ follows $\mathbf{P C}=\mathbf{X}$ and

$$
\mathbf{P}_{0}=\mathbf{X C}_{0}^{-1}=\mathbf{P C C}_{0}^{-1}=\mathbf{P} \mathbf{U}
$$

which leads by the orthogonality of $\mathbf{U}$ to $\mathbf{P}_{0} \mathbf{U}^{T}=\mathbf{P}$.

Theorem 1 and Theorem 2 show that the solutions of (A-1), (A-2) and (A-3) are given up to orthogonal transformations by $\mathbf{C}_{0}, \mathbf{S}_{0}$ and $\mathbf{P}_{0}$. An advantage of $\mathbf{C}_{0}$ and $\mathbf{S}_{0}$ compared to orthogonal transformations of those matrices lies in the fact that the singular value decomposition (SVD) of $\boldsymbol{\Phi}$ carries to some extent over to $\mathbf{C}_{0}$ and $\mathbf{S}_{0}$. To see this, let us denote the SVD of $\boldsymbol{\Phi}$ by:

$$
\boldsymbol{\Phi}=\mathbf{V}_{\Phi} \boldsymbol{\Sigma}_{\Phi} \mathbf{W}_{\Phi}^{T}
$$

where $\mathbf{V}_{\Phi}, \mathbf{W}_{\Phi} \in \mathbb{R}^{n \times n}$ are orthogonal matrices and $\boldsymbol{\Sigma}_{\Phi}=\operatorname{diag}\left(\sigma_{1}, \ldots, \sigma_{\mathrm{n}}\right)$ is a diagonal matrix, containing the singular values $\sigma_{i} \geq 0$ for $i=1, \ldots, n$ of $\boldsymbol{\Phi}$. Then, one easily recovers the following expressions due to the matrix square root properties as discussed below:

$$
\begin{aligned}
& \mathbf{C}_{0}=\mathbf{V}_{\Phi}\left(\mathbb{I}+\boldsymbol{\Sigma}_{\Phi}^{2}\right)^{-1 / 2} \mathbf{V}_{\Phi}^{T}, \\
& \mathbf{S}_{0}=\mathbf{V}_{\Phi}\left(\mathbb{I}+\boldsymbol{\Sigma}_{\Phi}^{-2}\right)^{-1 / 2} \mathbf{W}_{\Phi}^{T} .
\end{aligned}
$$

In the expressions above we have used that the matrix square root applied to the singular value decomposition of any symmetric matrix $\mathbf{C} \in \mathbb{R}^{n \times n}$ yields:

$$
\sqrt{\mathbf{C}}=\sqrt{\mathbf{U}_{C} \boldsymbol{\Sigma}_{C} \mathbf{U}_{C}^{T}}=\mathbf{U}_{C} \sqrt{\boldsymbol{\Sigma}_{C}} \mathbf{U}_{C}^{T}
$$

with $\mathbf{U}_{C} \in \mathbb{R}^{n \times n}$ being the orthogonal SVD matrix and the diagonal matrix $\boldsymbol{\Sigma}_{C}$ containing the singular values of $\mathbf{C}$. This result can be readily corroborated by:

$$
\mathbf{C}=\sqrt{\mathbf{C}} \sqrt{\mathbf{C}}=\mathbf{U}_{C} \sqrt{\boldsymbol{\Sigma}_{C}} \mathbf{U}_{C}^{T} \mathbf{U}_{C} \sqrt{\boldsymbol{\Sigma}_{C}} \mathbf{U}_{C}^{T}=\mathbf{U}_{C} \boldsymbol{\Sigma}_{C} \mathbf{U}_{C}^{T}
$$


Due to the property that the orthogonal SVD matrices are the same for any matrix and its matrix square root, the singular values of $\mathbf{C}=c(\mathbf{\Phi}), \mathbf{S}=s(\boldsymbol{\Phi})$ and $\mathbf{\Phi}$ can be linked directly and independently by trigonometric identities that relate in squares and square roots such as half-angle and double-angle formulae, and thus, allows to formulate analogies to these identities for the phase matrix $\mathbf{\Phi}$.

\section{APPENDIX B}

\section{DEFINITIONS OF AMPLITUDE AND PHASE ANISOTROPY}

In order to compare Amplitude and Phase Tensor parameter values, we need to define comparable anisotropy parameters for both tensors. In this work, we refer to anisotropy as macroscopic anisotropy as it is observed in measured impedance data at a certain period in contrast to microscopic anisotropy that can be considered in the subsurface model parameters. Further, we define two new parameters, the phase anisotropy, $\phi_{a}$, and the logarithmic amplitude anisotropy, $\rho_{a}$, which describe the anisotropy in the measured Phase and Amplitude Tensors with their singular values, $\phi_{1}$ and $\phi_{2}$, and $\rho_{1}$ and $\rho_{2}$, respectively:

$$
\phi_{a}=\frac{1}{2}\left(\arctan \phi_{1}-\arctan \phi_{2}\right) \quad \text { and } \quad \rho_{a}=\frac{1}{2}\left(\ln \rho_{1}-\ln \rho_{2}\right)
$$

and which can be compared like the real and imaginary parts of the logarithm of a complex number $z=\varrho \exp (i \varphi)$, that is $\ln z=\ln (\varrho \exp (i \varphi))=\ln \varrho+i \varphi$. Following this approach, we define $\rho$ and $\phi$ as:

$$
\rho=\sqrt{\rho_{1} \rho_{2}}=\exp \left(\frac{1}{2}\left(\ln \rho_{1}+\ln \rho_{2}\right)\right) \quad \text { and } \quad \phi=\frac{1}{2}\left(\arctan \phi_{1}+\arctan \phi_{2}\right)
$$


which represent the mean logarithmic amplitude and phase. For practical purposes the mean logarithmic amplitude can be scaled to represent apparent resistivity:

$$
\rho^{\mathrm{app}}=\frac{\rho^{2}}{\omega \mu_{0}},
$$

with angular frequency $\omega=2 \pi / T$, period $T$ and magnetic permeability of free space $\mu_{0}$. 


\section{REFERENCES}

Bibby, H. M., T. G. Caldwell, and C. Brown, 2005, Determinable and non-determinable parameters of galvanic distortion in magnetotellurics: Geophysical Journal International, 163 (3), 915-930.

Booker, J. R., 2014, The magnetotelluric phase tensor: A critical review: Surveys in Geophysics, 35 (1), 7-14.

Caldwell, T. G., H. M. Bibby, and C. Brown, 2004, The magnetotelluric phase tensor: Geophysical Journal International, 158 (2), 457-469.

Jones, A. G., 2012, Distortion of magnetotelluric data: its identification and removal, Chapter 6, in The Magnetotelluric Method: Theory and Practice: Cambridge University Press.

Martí, A., 2014, The role of electrical anisotropy in magnetotelluric responses: From modelling and dimensionality analysis to inversion and interpretation: Surveys in Geophysics, 35 (1), 179-218.

Patro, P. K., M. Uyeshima, and W. Siripunvaraporn, 2012, Three-dimensional inversion of magnetotelluric phase tensor data: Geophysical Journal International, 192 (1), 58-66.

Tietze, K., O. Ritter, and G. D. Egbert, 2015, 3-d joint inversion of the magnetotelluric phase tensor and vertical magnetic transfer functions: Geophysical Journal International, 203 (2), 1128-1148.

Weckmann, U., O. Ritter, and V. Haak, 2003, Images of the magnetotelluric apparent resistivity tensor: Geophysical Journal International, 155 (2), 456-468.

Weidelt, P., and A. D. Chave, 2012, The magnetotelluric response fuction, Chapter 4, in The Magnetotelluric Method: Theory and Practice: Cambridge University Press. 


\section{LIST OF FIGURE CAPTIONS}

Note that all figures are supplied in landscape format but are designed for a sideways setting. This allows for an easier comparison between left/right (Figure 1) and upper/lower (Figures 2 to 5) panels and it reduces the number of color pages to a minimum. The formatted height (sideways width) corresponds to Geophysics's one-and-a-third column (26 picas).

Figure 1: Decomposition parameters are plotted over periods for Phase, Amplitude, Apparent Resistivity Tensors (ART), and real and imaginary impedance tensors. The left hand side data corresponds to site J08 (inside the small anomaly, see Figure 2) and the right hand side data corresponds to site K11 (outside the small anomaly) in the model from Caldwell et al. (2004) described in the text.

Figure 2: Phase Tensor (a) and Amplitude Tensor (b) parameters at different periods (columns) obtained using the model from Caldwell et al. (2004). Tensor parameters are illustrated by scale (degrees and logarithmic apparent resistivity in color), anisotropy and strike angle (ellipse). Black rectangles reveal the location of the underlying conductive anomalies. 
Figure 3: Phase Tensor (a) and Amplitude Tensor (b) parameters at periods of $0.4 \mathrm{~s}$ (left) and $4 \mathrm{~s}$ (right) obtained using the 3D Modelling and Inversion Workshop (2016) Secret Model 3. Tensor parameters are illustrated by scale (degrees and logarithmic apparent resistivity in color), skew (circle fill - black: 3D, grey: quasi 2D, white: 2D), anisotropy and strike angle (ellipse). Orange ellipses locate identifiable anomalies that are described in the text.

Figure 4: Phase Tensor (a) and Amplitude Tensor (b) parameters at periods of $40 \mathrm{~s}$ (left) and 400s (right) obtained using the 3D Modelling and Inversion Workshop (2016) Secret Model 3. Tensor parameters are illustrated by scale (degrees and logarithmic apparent resistivity in color), skew (circle fill - black: 3D, grey: quasi 2D, white: 2D), anisotropy and strike angle (ellipse). Orange ellipses locate identifiable anomalies that are described in the text. 
Figure 5: Phase Tensor (a) and Amplitude Tensor (b) parameters at periods of 4,000 s (left) and 40,000s (right) obtained using the 3D Modelling and Inversion Workshop (2016) Secret Model 3. Tensor parameters are illustrated by scale (degrees and logarithmic apparent resistivity in color), skew (circle fill - black: 3D, grey: quasi 2D, white: 2D), anisotropy and strike angle (ellipse). Orange ellipses locate identifiable anomalies that are described in the text. 

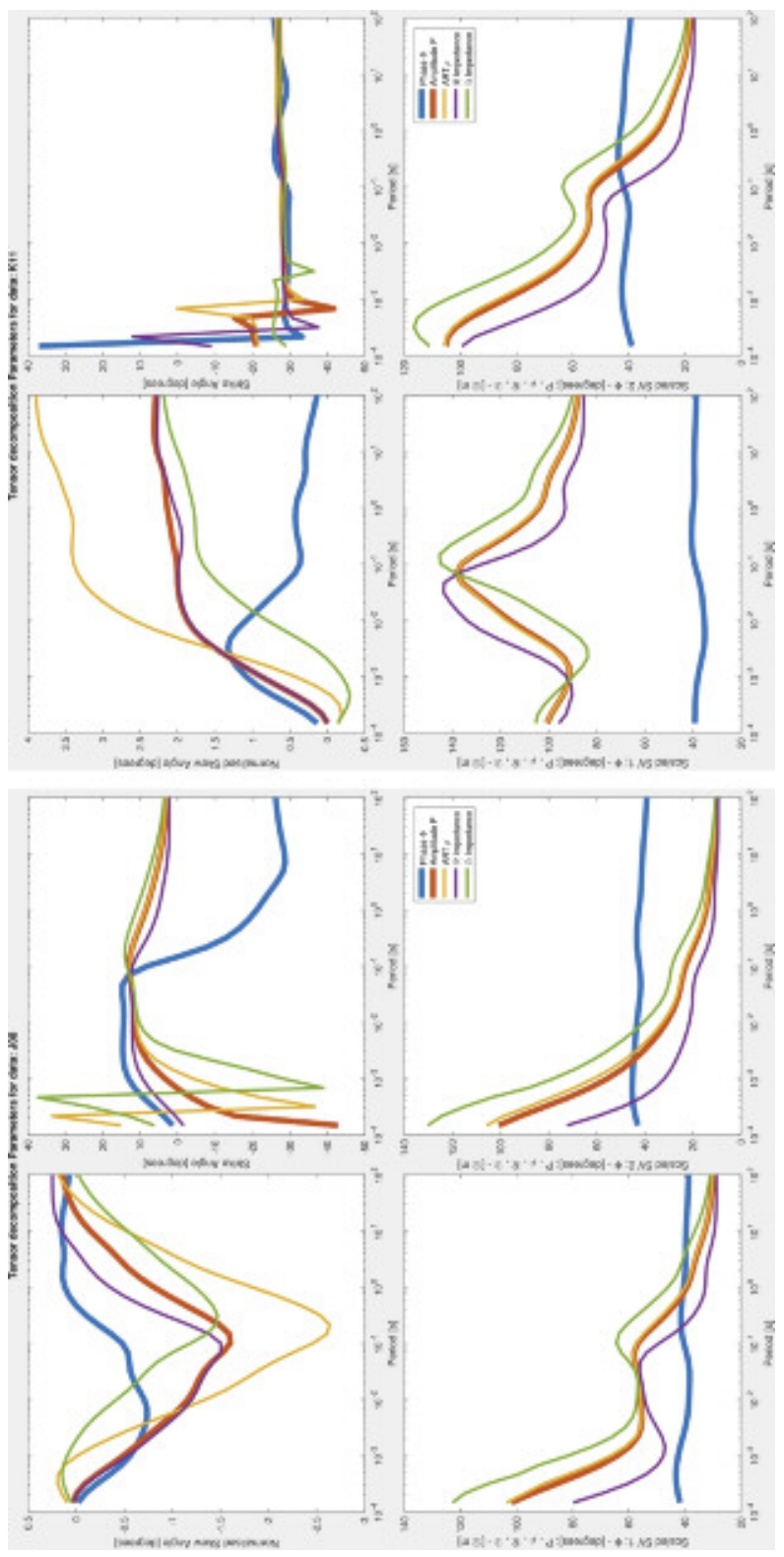

1-3 Decomposition parameters are plotted over periods for Phase, Amplitude, Apparent Resistivity Tensors (ART), and real and imaginary impedance tensors. The left hand side data corresponds to site 08 (inside the small anomaly, see Figure 2) and the right hand side data corresponds to site K11 (outside the small anomaly) in the model from Caldwell et al. (2004) described in the text.

\section{$54 \times 109 \mathrm{~mm}(600 \times 600 \mathrm{DPI})$}




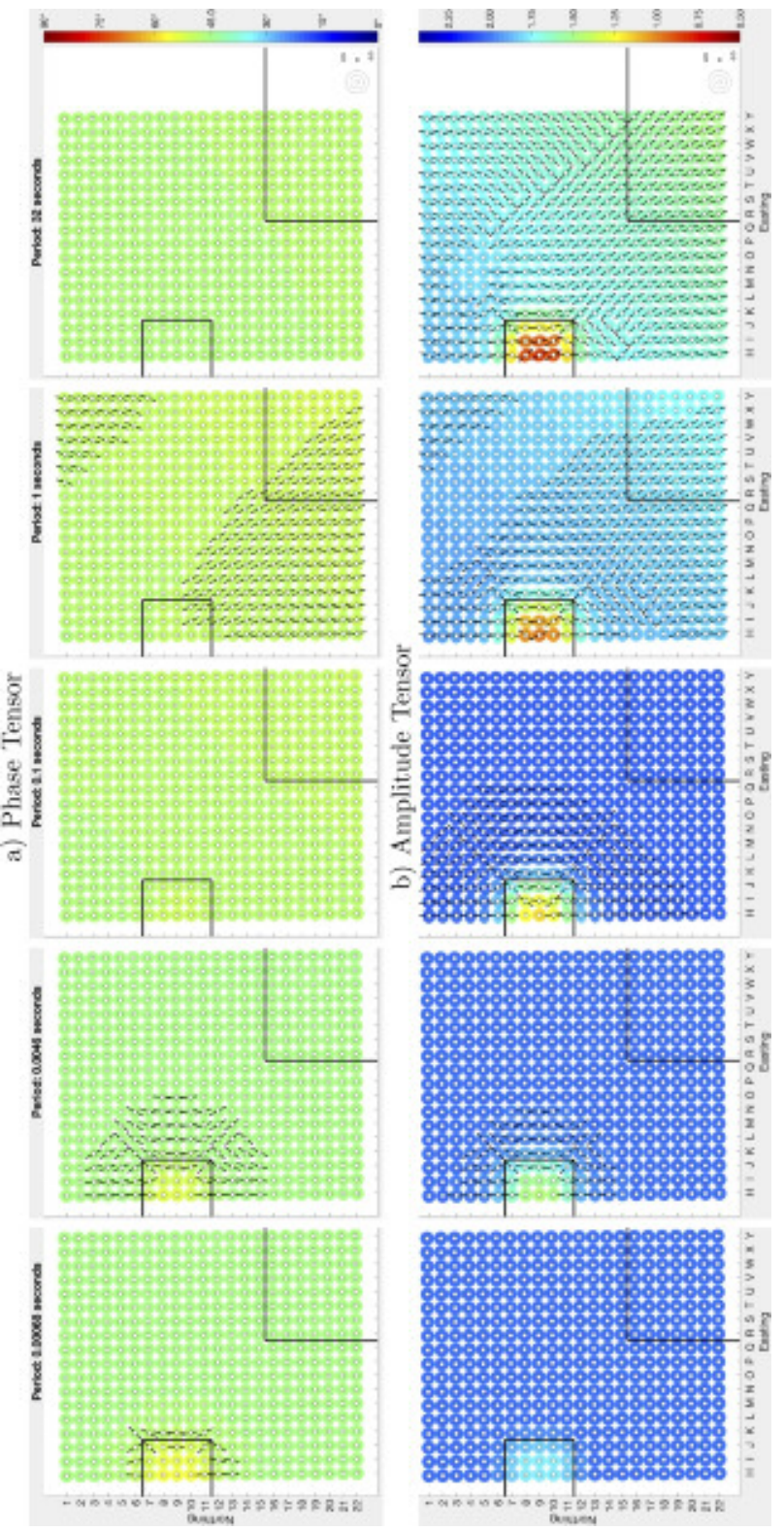

2 Phase Tensor (a) and Amplitude Tensor (b) parameters at different periods (columns) obtained using the model from Caldwell et al. (2004). Tensor parameters are illustrated by scale (degrees and logarithmic apparent resistivity in color), anisotropy and strike angle (ellipse). Black rectangles reveal the location of the underlying conductive anomalies.

$55 \times 109 \mathrm{~mm}(600 \times 600 \mathrm{DPI})$ 


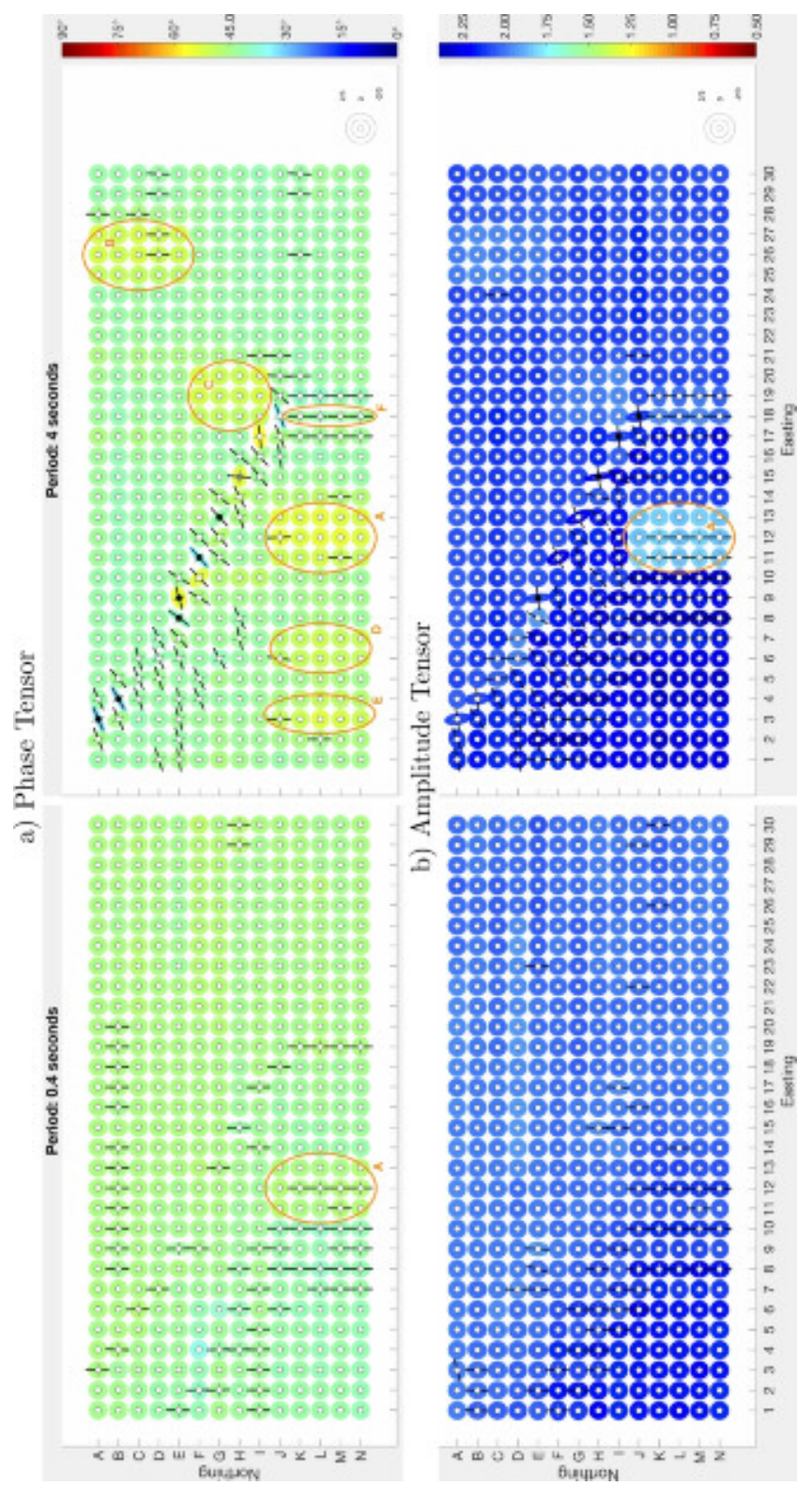

3 Phase Tensor (a) and Amplitude Tensor (b) parameters at periods of $0.4 \mathrm{~s}$ (left) and 4s (right) obtained using the 3D Modelling and Inversion Workshop (2016) Secret Model 3. Tensor parameters are illustrated by scale (degrees and logarithmic apparent resistivity in color), skew (circle fill - black: 3D, grey: quasi 2D, white: 2D), anisotropy and strike angle (ellipse). Orange ellipses locate identifiable anomalies that are described in the text.

$58 \times 109 \mathrm{~mm}(600 \times 600 \mathrm{DPI})$ 


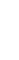

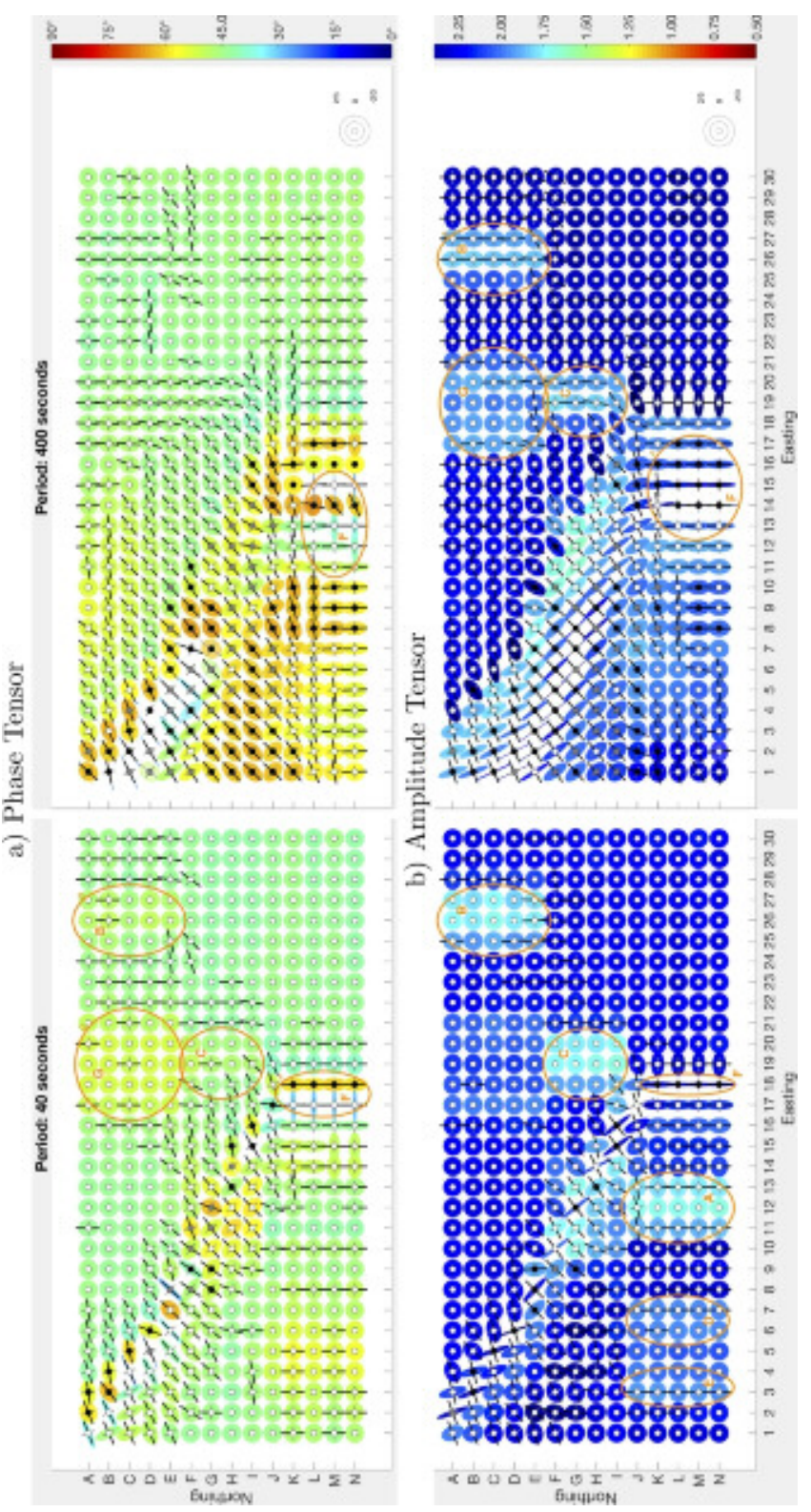

4 Phase Tensor (a) and Amplitude Tensor (b) parameters at periods of 40s (left) and 400s (right) obtained using the 3D Modelling and Inversion Workshop (2016) Secret Model 3. Tensor parameters are illustrated by scale (degrees and logarithmic apparent resistivity in color), skew (circle fill - black: 3D, grey: quasi 2D, white: 2D), anisotropy and strike angle (ellipse). Orange ellipses locate identifiable anomalies that are described in the text.

\section{$58 \times 109 \mathrm{~mm}(600 \times 600 \mathrm{DPI})$}




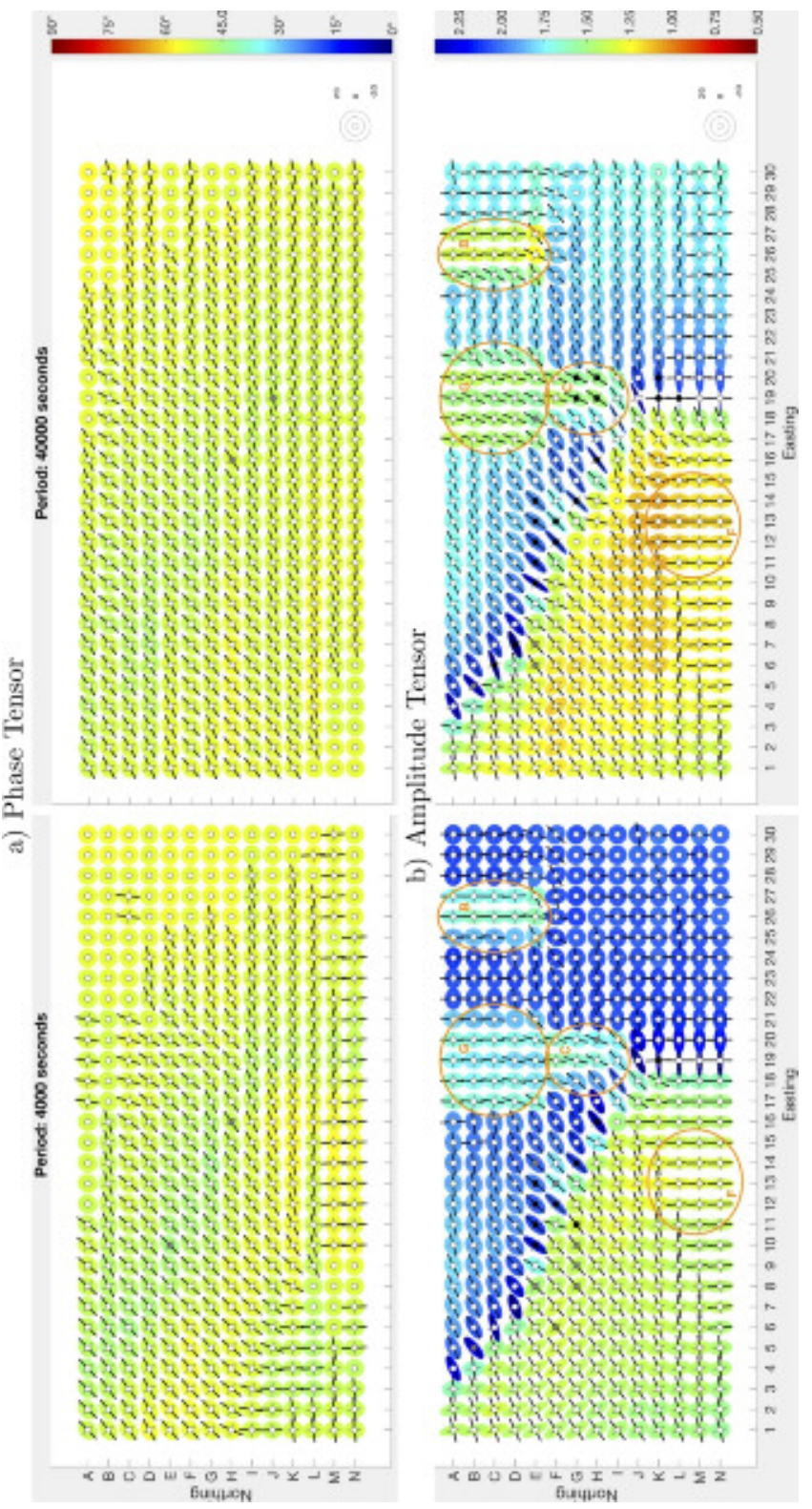

5 Phase Tensor (a) and Amplitude Tensor (b) parameters at periods of 4,000s (left) and 40,000s (right) obtained using the 3D Modelling and Inversion Workshop (2016) Secret Model 3. Tensor parameters are illustrated by scale (degrees and logarithmic apparent resistivity in color), skew (circle fill - black: 3D, grey: quasi 2D, white: 2D), anisotropy and strike angle (ellipse). Orange ellipses locate identifiable anomalies that are described in the text.

$58 \times 109 \mathrm{~mm}(600 \times 600 \mathrm{DPI})$ 
DATA AND MATERIALS AVAILABILITY

Data associated with this research are available and can be obtained by contacting the corresponding author. 